\title{
Design and Optimization of H.264 Encoder Based on the PNX1700 Platform
}

\author{
Zhengdong $\mathrm{Li}^{1, \mathrm{a},{ }^{*}, \text { Zhanle } \mathrm{Ma}^{2, \mathrm{~b}} \text {, Xiaobin Wang }}{ }^{3, \mathrm{c}}$, Yinxu He ${ }^{1, \mathrm{~d}}$, Chuan Peng ${ }^{1, \mathrm{e}}$ \\ ${ }^{1}$ Censtor of Simulation and Training, Chongqing Communication Institute, Chongqing, China \\ ${ }^{2}$ No.61516 of the People's Liberation Army, Beijing, China \\ ${ }^{3}$ Staff room of Communication tactics, Chongqing Communication Institute, Chongqing, China \\ agusi@foxmail.com, bzhanle@foxmail.com, cxiaobin@126.com, ${ }^{\mathrm{d}}$ yinxu@foxmail.com, \\ ppeng12@foxmail.com, \\ * corresponding author
}

Keywords: Code; Video; Platform; Process; Optimization

Abstract. The Nexperia PNX1700 Media Processor is a complete Audio/Video/Graphics system on a chip that contains a high-performance 32-bit VLIW processor, capable of high quality software video, audio signal processing. Based on Philips Semiconductor's multimedia processing chip Nexperia PNX1700, designs a new H.264 encoder with high performance for real time signal processing. Chooses the rapid integral discrete cosine translation and the advanced Hexagon searching algorithm, accomplishes the intelligent optimization and comprehensive debugging for codes. The results of experiments indicate that the encoder can encode all kinds of video data rapidly, and can realize the real time processing for all kinds of video signals.

\section{Introduction}

H.264 [1] is the new video standard project of the ITU-T Video Coding Experts Group (VCEG) and the ISO/IEC Moving Picture Experts Group (MPEG) [2]. In the standard, lots of new technology is invented, such as moment estimation with multi-modes, integer transformation of $4 \times 4$, united variable coding. H.264 has more enhanced compression performance and more significant improvement in the rate-distortion efficiency providing, typically, a factor of two in bit-rate savings when compared with existing standards such as the standard of MPEG-2 Video and H.263.

\section{Chip of PNX1700}

The PNX1700 [3] Media Processor Series is a complete Audio/Video/Graphics system on a chip that contains a high-performance 32-bit VLIW processor, TriMedia TM5250, capable of high quality software video (multi-video standard digital decoder/encoder and image improvement), audio signal processing, as well as general purpose control processing. It can either be used in standalone, or as an accelerator to a general purpose processor. The PNX1700 processes the input signals by utilizing several Audio/Video and co-processor modules before send them to the external peripherals. These modules provide additional video and data processing bandwidth without taking away precious CPU cycles. The combination of the CPU and co-processor modules makes the PNX1700 System On-Chip (SoC) suitable for most applications, especially those requiring high level of processing power/ throughput at a reduced cost.

\section{Optimization of H.264 encoder}

In order to realize the real time processing of video data, the H.264 encoder must be optimized across-the-board. According to the design principle of from top to down and modularization, the optimization can be realized from three layers, structure optimization, arithmetic optimization and code optimization [4]. 


\section{Structure optimization}

In view of the characteristic of PNX1700 and the model of H.264 standard, the encoder can be divided several modules: video input module, $4 \times 4$ integer transformation module, motion estimation module, image reconstruction module, quantization module, reverse quantization module, Zigzag scan module, Huffman encoding module. To make full advantage of the parallel processing of PNX1700, it is very important to reduce interim operations and avoid reduplicate manipulation as far as possible. For example, the integer transformation module, the integer inverse transformation module, the quantization module and the inverse quantization module, are close connected and can be regarded as one function. Accordingly, it avoids the problem that the coefficients are written into memory after the integer transformation module while they are fetched before the quantization module. According to the different characteristic of INTRA mode and INTER mode, the encoder is designed separately, which are showed in Figure 1 and Figure 2.

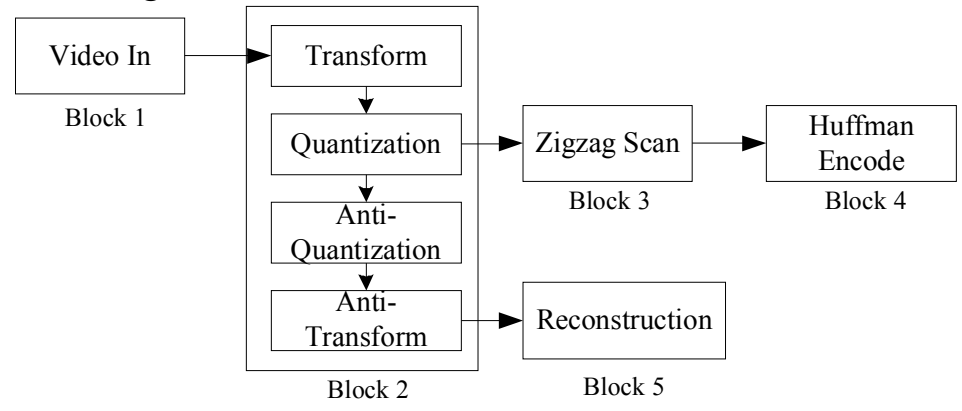

Figure 1. The structure design in INTRA mode

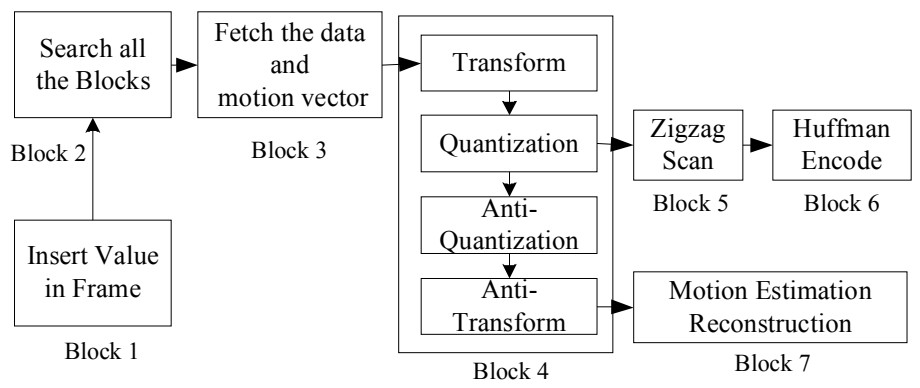

Figure 2. The structure design in INTER mode

\section{Arithmetic optimization}

In the H.264 encoder, the integer transformation module and the motion estimation module consume more time and resource of the processor than other modules. In order to enhance the performance of the encoder, it is indispensable to choose the highly effective fast transformation and the advanced search algorithm. They will be described in the next two sections.

\section{$4 \times 4$ integer transformation}

In the former standards, such as H.261, H.263, MPEG-4, $8 \times 8$ discrete cosine transformation (DCT) is taken as the basic transformation while $4 \times 4$ integer transformation is taken in H.264. The $4 \times 4$ unit not only reduces the computation quantity, but also reduces the joint errors between the Microblocks. Moreover, in the former standards, the real DCT has floating point calculation precision and often causes the drifting in the decoder. Therefore, H.264 reconstructs the transformation matrix and uses the integer DCT transformation, which effectively reduces the computation quantity, simultaneously keeps the precision of the video.

In order to enhance the operating speed of the transformation, on the one hand, the matrix multiplication operation can be transformed two unidimensional integers DCT transformation. On the other hand, the butterfly fast algorithm can be adopted to each unidimensional integer DCT transformation. The schematic drawing is showed in Figure 3. 


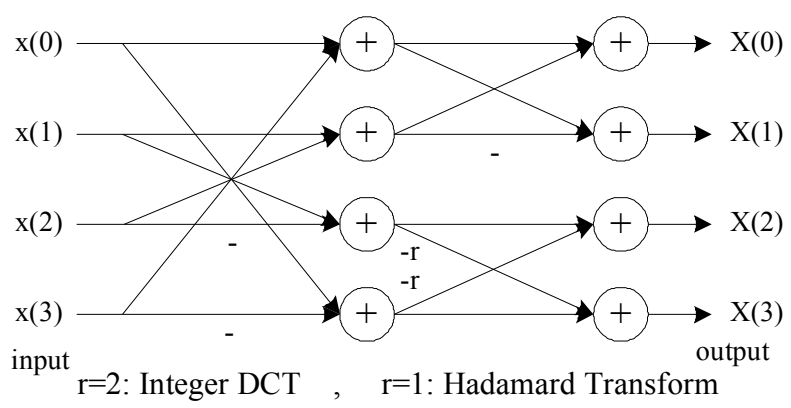

Figure 3. Unidimensional integer DCT transformation

\section{Motion estimation}

The motion estimate is most time-consuming module among all other modules [5]. Therefore, the rapid searching algorithm with high performance must be adopted. Comparing the current rapid searching algorithm: Three Step Search, Two-Dimensional Logarithmic, Cross Search Algorithm, Diamond Search, Hexagon Search [6], Hexagon Search is proved to be highest efficient among them. The schematic drawing of Hexagon Search is showed in Figure 4.
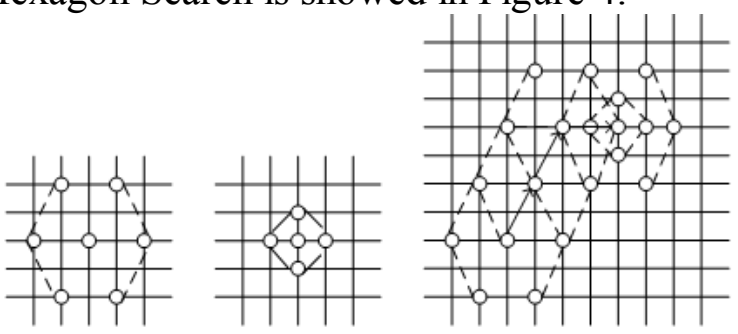

Figure 4. Hexagon search algorithm

Hexagon Search method combines Hexagonal Search mode and the small Diamond Search mode. Hexagonal Search mode has seven points: the center and the surrounding six points. The small Diamond Search mode has five points: the center and the vertical and horizontal four points. Firstly, in the Hexagon Search mode, the seven points around the center of the hexagon are searched and calculated. If the most excellent point is not the hexagonal center, the center of hexagon will be moved to this point. The Hexagon Search is repeated until the most excellent point is in its center. Secondly, the Hexagonal Search mode is switched to the small Diamond Search mode. According to search the five points around the diamond, the optimal point can be found ultimately.

\section{Code optimization}

\section{Loop unrolling}

Loop unrolling can enhance the parallel processing ability of the processor. Lots of cycle operations are used to process the video data matrixes in the encoder. Some functions also need the multistage nesting circulation. If there is not relation between the former data and the latter data, namely the current instruction result cannot be the following input, the method of loop unrolling may be used to process the parallel data. After the loop unrolling, five instructions-slots of the PNX1700 will be programmed efficiently and the parallel of instructions will be raised greatly.

\section{Custom operation}

In the structure of PNX1700, custom operation is aimed especially at the application of the multimedia. Suitable custom operation in the high language may enormously enhance the parallel of the processor. For example, when the Sum of Absolute Difference (SAD) is calculated, PNX1700 provides a special operation instruction: UME8UU. In this operation, one instruction may finish four subtraction operations, four absolution operations and three addition operations. Therefore, the reasonable use of custom operations will implement the parallel superiority of PNX1700 and enhance the operating speed of CPU. 


\section{Simulation results and discussions}

To test other standard CIF sequences: "Akiyo.yuv", "News.yuv", "Stefan.yuv" on the platform of PNX1700, the contrast parameters are shown in following Table 1.

As can be seen from the table, after the optimization, the frame rate of Stefan with cute movement surpasses 15f/s. Regarding the Foreman with large movement, the frame rate achieves $20 \mathrm{f} / \mathrm{s}$. When it comes to the Akiyo and the News with small movement, the frame rate may achieve about 30f/s. Therefore, the new H.264 encoder can continuously transmit the real-time data in the different environment.

Table 1. The comparison of the optimization

\begin{tabular}{ccccc}
\hline File(yuv) & Akiyo & News & $\begin{array}{c}\text { Forema } \\
\mathrm{n}\end{array}$ & Stefan \\
\hline Motion extent & tiny & small & large & acute \\
Before optimization (f/s) & 7.5 & 6.4 & 3.8 & 2.5 \\
After optimization (f/s) & 30.7 & 28.6 & 21.8 & 18.9 \\
Bit rate(kbps) & 155 & 264 & 380 & 1730
\end{tabular}

\section{Conclusions}

According to the H.264 standard model and the structure of the processor PNX1700, this article designs a new H.264 encoder with high performance for real time signal processing. Chooses the advanced rapid translation and searching algorithm, accomplishes the optimization and debugging for codes. The results of experiments indicate that the encoder can encode all kinds of video data rapidly, and can satisfy the real time processing of all kinds of video signals under all conditions.

\section{References}

[1] ITU-T Recommendation. H.264. Advanced video coding for generic audiovisual services 2003.5

[2] Bi Houjie, New Generation Standard of Video Compression-H.264/AVC, Beijing, Post \& Telecom Press, 2005.05

[3] Philips Semiconductors. PNX17xx Series Data Book. 2006.03

[4] Shen Lansun, Zhuo Li, Video Coding and Low Bit Transmission, Beijing Publishing House of Electronics Industry, 2001.12

[5] Yao Wang, Jörn Ostermann, Ya-Qin Zhang, Video Processing and Communications, Publishing House of Electronics Industry, 2003.6

[6] Ce Zhu, Xiao Lin and Lap-Pui Chau, "Hexagon-Based Search Pattern for Fast Block Motion Estimation", IEEE Trans. Circuits and Systems for Video Technology, Vol. 12(5), May.2002: 349-355. 